\title{
Development of a Novel One-Step Automated Rapid in situ Hybridization for Anaplastic Lymphoma Kinase Rearrangement Using Non-Contact Alternating-Current Electric-Field Mixing
}

\author{
Kazuhiro Imai ${ }^{a}$ Shinogu Takashima ${ }^{a}$ Satoshi Fujishima ${ }^{a}$ Tsubasa Matsuo $^{a}$ \\ Shin-nosuke Watanabe ${ }^{a}$ Hiroshi Nanjo $^{b}$ Yoichi Akagamic Ryuta Nakamurac \\ Kaori Terata ${ }^{a}$ Akiyuki Wakita ${ }^{a}$ Yusuke Sato $^{a}$ Satoru Motoyama ${ }^{a}$ \\ Yoshihiro Minamiya ${ }^{a}$ \\ ${ }^{a}$ Department of Thoracic Surgery, Akita University Graduate School of Medicine, Akita, Japan; ${ }^{b}$ Department of \\ Pathology, Akita University Hospital, Akita, Japan; ' Akita Prefectural Research and Development Center, Akita, Japan
}

\author{
Keywords \\ Lung cancer · Anaplastic lymphoma kinase · In situ \\ hybridization - Non-contact alternating-current \\ electric-field mixing
}

\begin{abstract}
Echinoderm microtubule-associated protein-like 4 and anaplastic lymphoma kinase $(A L K)$ fusion gene rearrangement is a key driver mutation in non-small cell lung cancer (NSCLC). Although Break-Apart $A L K$ fluorescence in situ hybridization (FISH) is a reliable diagnostic method for detecting ALK gene rearrangement, it is also costly and time-consuming to use as a routine screening test. Our aim was to evaluate the clinical utility of a novel one-step, automated, rapid FISH (AutoRaFISH) method developed to facilitate hybridization. This method takes advantage of the non-contact mixing effect of an alternating-current electric field. Ten representative specimens from 85 patients diagnosed at multiple centers with primary lung cancer with identified $A L K$-FISH status were collected. The specimens were all tested using FISH, RaFISH,
\end{abstract}

and Auto-RaFISH. With both RaFISH protocols, the ALK test was completed within $4.5 \mathrm{~h}$, as compared to the $20 \mathrm{~h}$ needed for the standard protocol. We found $100 \%$ agreement between the standard FISH, RaFISH, and new Auto-RaFISH based on the $A L K$ status, and all methods stained equally well. These findings suggest that Auto-RaFISH could potentially serve as an automated clinical tool for prompt determination of $A L K$ status in NSCLC.

(c) 2020 S. Karger AG, Basel

\section{Introduction}

Several key genetic alterations drive non-small cell lung cancer (NSCLC). These include mutation of the epidermal growth factor receptor (EGFR) gene and rearrangement of the echinoderm microtubule-associated protein-like 4 and anaplastic lymphoma kinase (EML4$A L K)$ fusion gene [1-5]. The EML4-ALK fusion gene is one of the most desirable molecular targets for patients with $A L K$-rearranged NSCLC because there are several

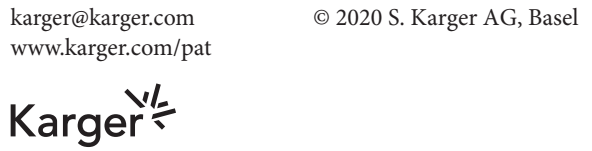

Kazuhiro Imai, $\mathrm{MD}, \mathrm{PhD}$

Department of Thoracic Surgery

Akita University Graduate School of Medicine

1-1-1 Hondo, Akita 010-8543 (Japan)

i-karo@mui.biglobe.ne.jp 
specific tyrosine kinase inhibitors (TKIs) available for treatment [1], including crizotinib, ceritinib, alectinib, brigatinib, and lorlatinib [6,7]. Moreover, ALK TKIs are well tolerated and induce rapid, durable responses in $A L K$-positive patients, and there is potential for ongoing benefit after initial disease progression in this population $[6,8]$. It is therefore widely recognized that the detection of $A L K$ gene rearrangements is a very important determinant when selecting an effective chemotherapy for patients with advanced and/or recurrent NSCLC.

Because $A L K$ gene rearrangement involves large chromosomal inversion and translocation, fluorescence in situ hybridization (FISH) has become the method of choice for detecting all forms of $A L K$ gene rearrangement, including the rare non-EML4 fusions, and it was the assay used to detect this genetic aberration in the first clinical trials of $A L K$ inhibitor therapy with crizotinib [811]. As many guidelines already recommend, BreakApart ALK FISH is a reliable standard diagnostic method in surgical pathology because it is easily applicable to formalin-fixed, paraffin-embedded samples [5]. However, FISH is time-consuming and requires the use of expensive probes and a special fluorescence microscopy facility. This means that several obstacles to cost-effective and timely screening must be overcome before FISH is introduced as a routine test.

We have been developing a rapid immunohistochemistry (IHC) method that makes use of an alternating-current (AC) electric field to facilitate the antigen-antibody reaction. With this device, we apply a high-voltage, low(or high-) frequency AC electric field to the tissue sections. The antibody is mixed within microdroplets as the voltage is switched on and off at specific intervals. The resultant Coulomb force stirs the diluted solution on the sections, which increases the opportunity for contact between the antigen and antibody. This rapid-IHC method enables prompt detection of target cells in frozen sections and can provide a surgeon with an intraoperative diagnosis within $30 \mathrm{~min}$, as opposed to the 3-6 h required for conventional IHC [12-16]. In addition, we have shown that this technique is potentially applicable to multiple assays, for example in the hybridization step of FISH. We previously reported its usefulness for ISH-human EGFR 2 (HER2) in breast cancer and FISH-ALK rearrangement in lung cancer [17-19]. With rapid FISH using non-contact AC electric mixing (RaFISH), the ALK test was completed within $4.5 \mathrm{~h}$, as compared to $20 \mathrm{~h}$ needed for the standard FISH. Moreover, there was $97.6 \%$ agreement between the standard FISH and RaFISH based on the ALK signal status (Cohen's kappa coefficient $=0.904,95 \%$ con- fidence interval: 0.802-1.000). Thus, while RaFISH produced results more promptly than standard FISH, staining and the accuracy of the $A L K$ evaluation achieved with RaFISH was equal to the standard [18].

RaFISH thus has the potential for use as a clinical tool enabling prompt determination of $A L K$ status. However, a limitation of RaFISH is that it is subject to human error because the ISH steps are manually performed. To address this limitation, we developed a new automated RaFISH (Auto-RaFISH), which reduces the effort of researchers or technicians and stabilizes the quality of the $A L K$ rearrangement assay. The aim of the present methods article was to evaluate the clinical utility and reliability of this new Auto-RaFISH technique.

\section{Methods and Design}

\section{Patients and Specimens}

A total of 10 representative samples were adequately collected from enrolled lung cancer patients described in our earlier report [18]. These included 4 of the 19 EML4-ALK-positive samples collected for IHC and FISH at the Akita University Hospital, Akita Red Cross Hospital, Omagari Kousei Medical Center, and Nakadori General Hospital between April 2008 and December 2016, plus 6 of the 66 negative $/ 1+(2+)$ samples collected for study between January 2015 and December 2016 at Akita University Hospital. Using standard histological techniques, the specimens were fixed in $10 \%$ formalin, embedded in paraffin, cut at 3-4 $\mu \mathrm{m}$, transferred to slides, and stained using hematoxylin/eosin and IHC. In addition, the specimens were subjected to FISH and our AutoRaFISH protocol.

\section{Standard IHC}

Anti-ALK IHC was performed using the iAEP method (ALK Detection Kit; Nichirei Bioscience, Tokyo, Japan) with an automatic staining protocol $[20,21]$. To confirm $A L K$ positivity, IHC (and/or FISH) was also performed and evaluated by a commercial clinical laboratory (SRL, Tokyo). ALK IHC results were classified using iScore for iAEP IHC $[22,23]$.

\section{Standard FISH}

Standard FISH was performed on unstained 4- $\mu \mathrm{m}$ sections of $10 \%$ formalin-fixed, paraffin-embedded tumor tissue using an ALK Break-Apart probe set (Vysis ALK Break Apart FISH Probe kit; Abbott Molecular Inc., IL, USA) with a paraffin pretreatment reagent kit (Vysis Paraffin Pretreatment IV and Post-Hybridization Wash Buffer kit; Abbott Molecular Inc., IL, USA). Assays were performed following the manufacturer's instructions.

\section{Non-Contact AC Electric-Field Mixing}

It is known that when an AC electric field is applied to a solution, electro-osmotic vortices are produced to induce non-contact mixing. We have been ever developing a rapid-IHC method that makes use of an AC electric field to facilitate the antigen-antibody reaction. The rapid-IHC device mounts with the temperature control unit and equipped with a humidifier to prevent evaporation of
Imai et al. 
Table 1. Procedural details for standard FISH and RaFISH

\begin{tabular}{llll}
\hline Protocol & Standard & RaFISH & Auto-RaFISH \\
\hline Dewaxing, activation, dehydration & $1 \mathrm{~h}$ & $1 \mathrm{~h}$ & $1 \mathrm{~h}$ \\
Denaturation and hybridization & $16-18 \mathrm{~h}\left(37^{\circ} \mathrm{C}\right)$ & $3 \mathrm{~h}\left(37^{\circ} \mathrm{C}\right), \mathrm{AC}$ & $3 \mathrm{~h}\left(37^{\circ} \mathrm{C}\right), \mathrm{AC}$ \\
Washing slides, etc. & $10 \mathrm{~min}$ & $10 \mathrm{~min}$ & $\begin{array}{l}\text { (+drop oil cover }) \\
\text { Microscopy after DAPI stain }\end{array}$ \\
Total required time & $10 \mathrm{~min}$ & $10 \mathrm{~min}$ & $4.5 \mathrm{~h}$ \\
\hline
\end{tabular}

DAPI, 4',6-diamidino-2-phenylindole dihydrochloride; AC, alternating-current electric field (4.5 kv, 90 [or 15] $\mathrm{Hz})$.

the microdroplet. The microscope slides can be put between the electrodes. A high voltage $\left(4.5 \mathrm{kV}_{\mathrm{p} \text {-p }}\right.$, offset $\left.2.25 \mathrm{kV}\right)$, low-high frequency $(15-90 \mathrm{~Hz})$ AC electric field was applied to the sections. The device features an air insulation layer between the upper electrode and microdroplet. Relative permittivity of air insulation and microdroplet (when the droplet is pure water) is $\varepsilon a=1$ and $\varepsilon r=80$, respectively. An attractive force mainly acts on the microdroplet and induces positive electric-field direction by the difference between relative permittivity $(\varepsilon \mathrm{a}<\varepsilon r)$. Even if the stirrer is not in the droplet, the reagent (antibody or RNA/DNA probe) is mixed within the microdroplet as the voltage is switched on and off in a time series $(\mathrm{I} \rightarrow \mathrm{II} \rightarrow \mathrm{III} \rightarrow \mathrm{IV}$, online suppl. Fig. 1; for all online suppl. material, see www.karger.com/doi/10.1159/000505631). The resultant Coulomb force stirs the reagent solution on the sections, and the opportunity for contact between the reagent and the gene is increased because as the voltage is turned on and off at regular intervals, the microdroplet's shape is transformed. If high-frequency AC electric field is applied, the shape is almost not transformed. However, a high-speed camera can observe the imperceptible transformation, and it can stir the reagents.

\section{New RaFISH and One-Step Auto-RaFISH}

We have developed a prototype for Auto-RaFISH (Fig. 1). The theory behind the technique for AC electric-field mixing and its protocol were also described in detail previously [17-19]. Ten microliters of Vysis LSI ALK Dual Color Break-Apart FISH Probe and $40 \mu \mathrm{L}$ of sterile distilled water were applied to each slide, after which $40 \mu \mathrm{L}$ of HAIKORU-K140N (KANEDA, Japan), a very lowviscosity liquid paraffin $\left(4.0-5.5 \mathrm{~mm}^{2} / \mathrm{s}\right)$, were added as an oil cover to prevent probe evaporation. The slide was then placed between electrode plates, and a high-voltage $\left(4.5 \mathrm{kV}_{\mathrm{p}-\mathrm{p}}\right.$, offset $\left.2.25 \mathrm{kV}\right)$, high-frequency $(90 \mathrm{~Hz}) \mathrm{AC}$ was applied after denaturation for $3 \mathrm{~min}$ at $73^{\circ} \mathrm{C}$. There was a distance of $3.8-4.0 \mathrm{~mm}$ between the slide and electrode plates, and the current was applied for $3 \mathrm{~h}$ at $37^{\circ} \mathrm{C}$. After washing the slide, $10 \mu \mathrm{L}$ of DAPI (4',6-diamidino2-phenylindole dihydrochloride) counterstain were applied. Signals were analyzed using an Olympus BX51 fluorescence microscope (Olympus, Tokyo, Japan). A minimum of 50 nuclei from two separate areas of the tumor were independently scored by two technologists. The run protocol for Auto-RaFISH was established so that the entire assay procedure, consisting of pretreatment, hybridization, stringency wash, and signal detection, was completed as a one-step automated assay. Only the oil cover step was performed manually. Table 1 summarizes each procedure and the time required for conventional FISH, RaFISH, and Auto-RaFISH.

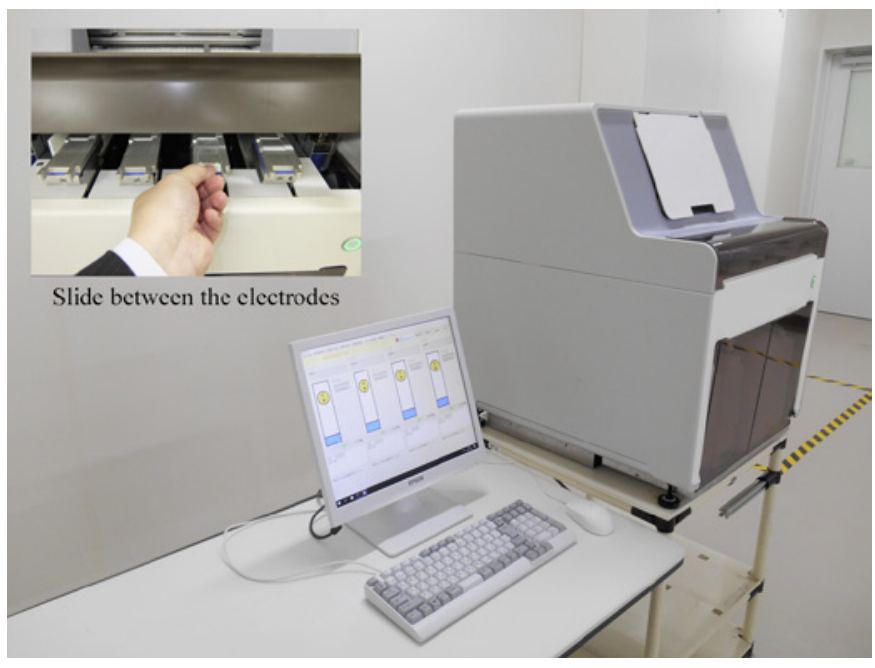

Fig. 1. One-step Auto-RaFISH device used to apply a high-voltage, low-frequency, AC electric field. The new device automatically performs the denaturation, hybridization, and slide-washing steps.

\section{Statistical Analysis}

Statistical analysis was performed using JMP IN 11.0.0 software (SAS Institute, Cary, NC, USA). Cohen's kappa coefficient was used to assess agreement of $4 \times 2$-contingency tables between protocols. A kappa value $<0.4$ indicated fair-to-poor agreement, $0.4-$ 0.8 indicated moderate-to-good agreement, and $>0.8$ indicated excellent agreement.

Detection of ALK Rearrangement Using New Auto-RaFISH

Using the procedures outlined in Table 1, the new Auto-RaFISH enabled detection of $A L K$ rearrangement within $4.5 \mathrm{~h}$, as compared to $18-20 \mathrm{~h}$ needed for standard FISH. In ALK-positive patients, the performances of the new Auto-RaFISH protocol, RaFISH, and standard FISH were equal (Cases 1-4, Fig. 2). Online supplementary Figure 2 shows typical $A L K$ rearrangement-positive and -negative cells detected by Auto-RaFISH. All 10 samples were well stained and accurately evaluated. Table 2 shows the $A L K$ statuses determined from the $A L K$ signal for all samples. We found $100 \%$ agreement between conventional FISH, RaFISH, and Auto-RaFISH based on the status of the $A L K$ signals in the 10 representative samples. 

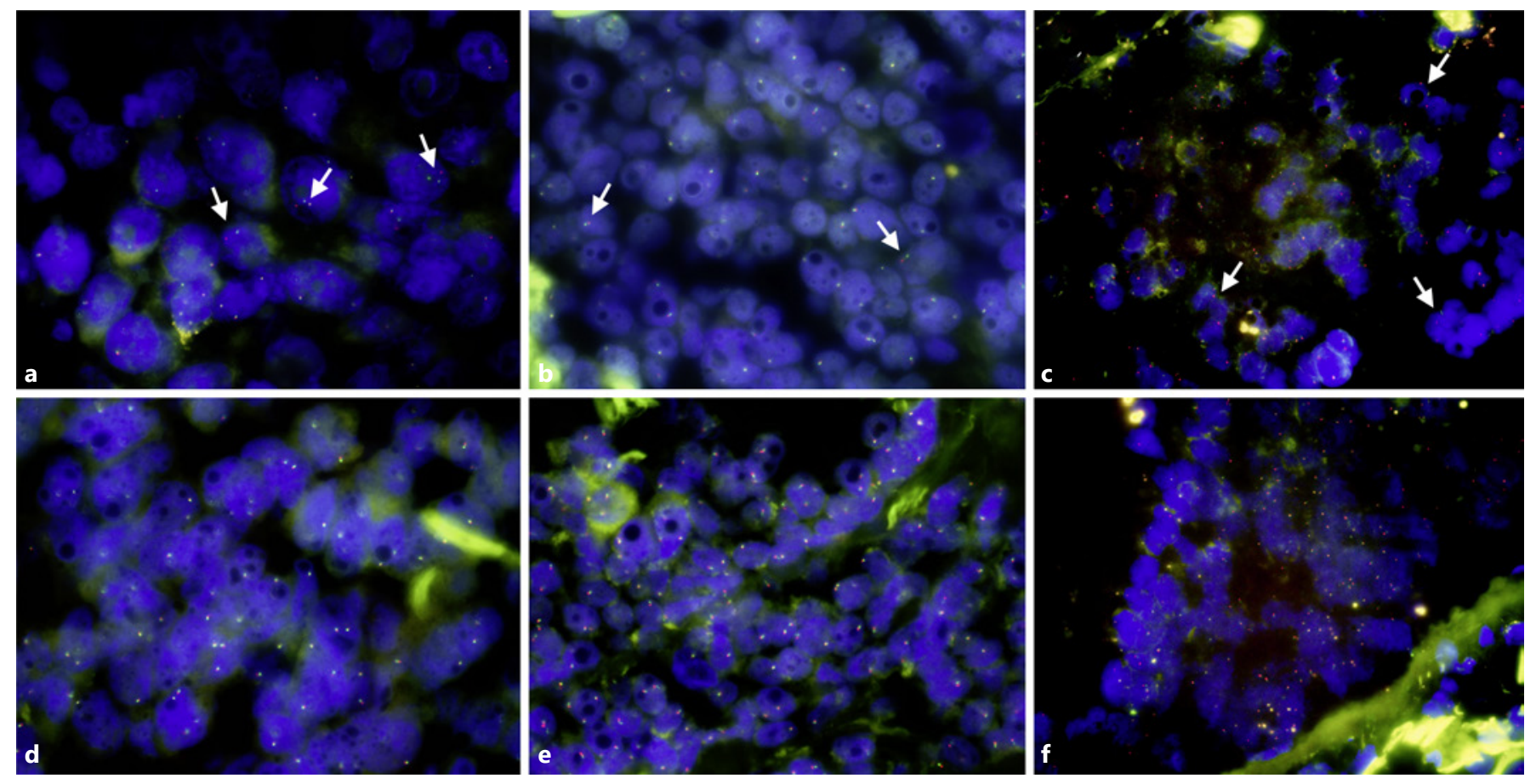

Fig. 2. Detection of $A L K$ rearrangement using new Auto-RaFISH. a Positivity demonstrated using conventional FISH. b Positivity demonstrated using RaFISH. c Positivity demonstrated using Auto-RaFISH. d Negativity demonstrated using conventional FISH. e Negativity demonstrated using RaFISH. f Negativity demonstrated using Auto-RaFISH. Samples were classified as positive for

Table 2. Positive $A L K$ rearrangement determined using standard FISH, RaFISH, and one-step Auto-RaFISH

\begin{tabular}{clll}
\hline Sample & Standard FISH & RaFISH & Auto-RaFISH \\
\hline 1 & + & + & + \\
2 & + & + & + \\
3 & + & + & + \\
4 & + & + & + \\
5 & - & - & - \\
6 & - & - & - \\
$7 *$ & - & - & - \\
8 & - & - & - \\
9 & - & - & - \\
10 & - & - & \\
\hline
\end{tabular}

-, no stain; +, rearrangement. Results were evaluated by two independent pathologists in accordance with the new guideline recommendations. * Sample from another tumor location in the same patient from whom sample 6 was collected. Auto-RaFISH, automated FISH with AC mixing.
$A L K$ rearrangement when $\geq 15 \%$ of the tumor nuclei showed split signals (red and green signals were separated by $\geq 2$ signal diameters, white arrows) or single red signals $\left(3^{\prime} A L K\right)$ were observed in FISH or RaFISH. Yellowish cytoplasmic staining in the tumor cells shows tissue collagen.

\section{Discussion}

In the present methods article, we demonstrated that our new Auto RaFISH, performed with a high-voltage, high-frequency AC electric field, can be used to detect $A L K$ rearrangement within $4.5 \mathrm{~h}$ and can be completed as a one-step automated assay. The Auto-RaFISH and standard FISH stained nearly equally well in patients with $A L K$-positive lung cancer.

The ALK Break-Apart FISH Probe Kit has become the gold standard diagnostic tool for detecting $A L K$ rearrangements to determine the appropriateness of targeted therapy with ALK inhibitors in NSCLC [5]. However, the FISH protocol has several well-known disadvantages: it is time-consuming, technically demanding, costly, and may delay final determination of $A L K$ status. The standard FISH protocol requires approximately $20 \mathrm{~h}$ to complete, as hybridization usually takes $16-18 \mathrm{~h}$. By contrast, RaFISH enabled the hybridization step to be completed in less than $3 \mathrm{~h}$. However, because all steps in the RaFISH protocol except hybridization are performed manually, the method is subject to human error and needed im- 
provement. For this series, therefore, we developed a onestep Auto-RaFISH device, which stabilizes test quality.

We previously reported the usefulness of AC mixing for detection of lung cancer metastasis, breast cancer metastasis, central nervous system tumors, and mammalian eggs, as well as for ISH-HER2 and ISH-EML4-ALK rearrangement in lung cancer [12-19]. New ISH with AC mixing is summarized in Table 3. The AC mixing device can also be applied to rapid ISH $[17,18]$ and reagentsaving ISH [19]. Auto-RaFISH of ALK in the present study did not have the costly advantage because the same amount of Vysis LSI ALK Dual Color Break-Apart FISH Probe was used in both new RaFISHs and the standard FISH. Importantly, in our earlier report [19], ISH with AC mixing can be used with 1:2 saving ISH to accurately diagnose HER2 status and achieve stable HER2 gene hybridization in breast cancer samples, even when reagent concentrations vary. Although ISH with AC mixing produces results more promptly while using smaller amounts of probe, staining and the accuracy of the target gene status evaluation with ISH with AC mixing may be equal to or greater than with standard ISH. These results suggest that new ISH with AC mixing could be used as a clinical tool to promptly determine target gene status.

However, AC mixing ISH has some limitations. First, ISH usually requires a warm temperature $\left(37^{\circ} \mathrm{C}\right.$ for hybridization overnight and $80^{\circ} \mathrm{C}$ for denaturation) and a longer processing time than the rapid IHC method. Consequently, evaporation could be a problem. We therefore developed a very-low-viscosity liquid paraffin to be used as an oil cover with the Ra-FISH device. Second, because pores created when an electric current is applied to the cell membrane could cause false positives and overdiagnosis, it was important to consider the effect of electroporation when using the $\mathrm{AC}$ mixing device for IHC or ISH. In our earlier report, we showed that the electric current does not flow through the inside of microdroplets during AC electric-field mixing [14]. This is because the stirred microdroplet is separated from the electrode by both glass and air, which act as insulators. Consequently, no pores are created. Third, the new Auto-RaFISH device is currently still in development. In particular, the automatic washing step did not work in some cases because the washing pressure was compensated for. The important fourth limitation of this study includes the small sample size and possible selection bias, as the prototype under development was used in the present study. Future research is needed to provide the additional data required to complete this product. Fifth, specimens in the present study sometimes could not be evaluated using the RaFISH protocol because
Table 3. New ISH with AC mixing

\begin{tabular}{lllll}
\hline First author [ref], year & $\begin{array}{l}\text { Target } \\
\text { gene }\end{array}$ & $\begin{array}{l}\text { Probe } \\
\text { dilution }\end{array}$ & $\begin{array}{l}\text { Protocol } \\
\text { time }\end{array}$ & $\begin{array}{l}\text { Kappa } \\
\text { value }\end{array}$ \\
\hline Saito [17], 2016 & HER2 & $1: 1$ & $6 \mathrm{~h}$ & 0.960 \\
Fujishima [18], 2017 & ALK & $1: 1$ & $4.5 \mathrm{~h}$ & 0.904 \\
Kurihara [19], 2019 & HER2 & $1: 2^{*}$ & $19-21 \mathrm{~h}$ & 0.912 \\
\hline
\end{tabular}

ISH with AC mixing could be used as a clinical tool for prompt and stable determination of target gene status, even when reagent concentrations vary. * Practical application of AC mixing reagentsaving ISH.

of poor $A L K$ hybridization, which was caused by there being too much collagen tissue on the specimens. However, it is a common problem for $A L K$-FISH.

\section{Conclusion}

In summary, we have presented a new one-step automated FISH (Auto-RaFISH) technology that could potentially serve as a clinical tool for prompt determination of $A L K$ status in lung cancer samples. The clinical benefit of time-effective Auto-RaFISH is that $A L K$-positive NSCLC patients can receive specific TKIs therapy more promptly, if the patients have the result of Break-Apart ALK-FISH at the same time of diagnostic biopsy. Although the present study is preliminary, with only a small sample size and without an optimized ALK RaFISH protocol, our findings indicate that this approach warrants further investigation.

\section{Acknowledgement}

The authors are grateful to Mr. Iwao Shimizu (Sakura Finetek Japan Co., Ltd, Tokyo, Japan) and Mr. Junya Enomoto and Mr. Taro Aoki (EPSON, Akita, Japan) for advice on the principles of AC electric-field mixing and development of the new Auto-RaFISH device. We also thank Prof. Shinya Tanaka of the Department of Cancer Pathology, Hokkaido University, who provided advice on ISH.

\section{Statement of Ethics}

All experimental protocols were approved by the institutional review board at Akita University Hospital (Permit No. 1408), and written informed consent was obtained from all patients. The methods used in this study were carried out in accordance with approved guidelines. 


\section{Disclosure Statement}

The authors have no conflicts of interest to disclose.

\section{Author Contributions}

K.I. analyzed the data and wrote the paper. S.T., S.F., T.M., S.W., K.T., A.W., Y.S., and S.M. helped collect the data. H.N. made pathological diagnoses and supported this research. R.N. and Y.A. contributed to the development of the device enabling application of a high-voltage, low-frequency AC electric field. Y.M. designed and supervised the research. All authors read and approved the final manuscript.

\section{References}

1 Soda M, Choi YL, Enomoto M, Takada S, Yamashita $Y$, Ishikawa $S$, et al. Identification of the transforming EML4-ALK fusion gene in non-small-cell lung cancer. Nature. 2007 Aug;448(7153):561-6.

2 Rikova K, Guo A, Zeng Q, Possemato A, Yu J, Haack $H$, et al. Global survey of phosphotyrosine signaling identifies oncogenic kinases in lung cancer. Cell. 2007 Dec;131(6):1190-203.

3 Tsao AS, Scagliotti GV, Bunn PA Jr, Carbone DP, Warren GW, Bai C, et al. Scientific Advances in Lung Cancer 2015. J Thorac Oncol. 2016 May;11(5):613-38.

4 Hiley CT, Le Quesne J, Santis G, Sharpe R, de Castro DG, Middleton G, et al. Challenges in molecular testing in non-small-cell lung cancer patients with advanced disease. Lancet. 2016 Sep;388(10048):1002-11.

5 Tsao MS, Hirsch FR, Yatabe Y, editors. IASLC atlas of ALK testing in lung cancer. Aurora (CO): IASLC Press; 2013.

6 Matikas A, Kentepozidis N, Georgoulias V, Kotsakis A. Management of Resistance to Crizotinib in Anaplastic Lymphoma KinasePositive Non-Small-cell Lung Cancer. Clin Lung Cancer. 2016 Nov;17(6):474-82.

7 Hallberg B, Palmer RH. The role of the ALK receptor in cancer biology. Ann Oncol. 2016 Sep;27 Suppl 3:iii4-15.

8 Camidge DR, Bang YJ, Kwak EL, Iafrate AJ, Varella-Garcia M, Fox SB, et al. Activity and safety of crizotinib in patients with ALK-positive non-small-cell lung cancer: updated results from a phase 1 study. Lancet Oncol. 2012 Oct;13(10):1011-9.

9 Blackhall F, Cappuzzo F. Crizotinib: from discovery to accelerated development to frontline treatment. Ann Oncol. 2016 Sep;27 Suppl 3:iii35-41.
10 Bang Y, Kwak EL, Shaw AT, Camidge DR, Iafrate AJ, Maki RG, et al. Clinical activity of the oral ALK inhibitor PF-02341066 in ALK-positive patients with non-small cell lung cancer (NSCLC). J Clin Oncol. 2010;28: suppl; abstr 3.

11 Kwak EL, Bang YJ, Camidge DR, Shaw AT, Solomon B, Maki RG, et al. Anaplastic lymphoma kinase inhibition in non-small-cell lung cancer. N Engl J Med. 2010 Oct;363(18):1693-703.

12 Toda H, Minamiya Y, Kagaya M, Nanjo H, Akagami Y, Saito H, et al. A novel immunohistochemical staining method allows ultrarapid detection of lymph node micrometastases while conserving antibody. Acta Histochem Cytochem. 2011 Jun;44(3):133-9.

13 Tanino M, Sasajima T, Nanjo H, Akesaka S, Kagaya M, Kimura T, et al.; R-IHC Study Group. Rapid immunohistochemistry based on alternating current electric field for intraoperative diagnosis of brain tumors. Brain Tumor Pathol. 2015 Jan;32(1):12-9.

14 Shirasawa H, Kumagai J, Sato E, Kabashima K, Kumazawa Y, Sato W, et al. Novel method for immunofluorescence staining of mammalian eggs using non-contact alternating-current electric-field mixing of microdroplets. Sci Rep. 2015 Oct;5(1):15371.

15 Terata K, Saito H, Nanjo H, Hiroshima Y, Ito S, Narita K, et al. Novel rapid-immunohistochemistry using an alternating current electric field for intraoperative diagnosis of sentinel lymph nodes in breast cancer. Sci Rep. 2017 Jun;7(1):2810.

16 Hoshino I, Imai K, Nanjo H, Nakamura R, Saito Y, Fujishima S, et al. Reagent-saving immunohistochemistry for HER2 using noncontact alternating current electric field mixing. J Clin Pathol. 2019 Jan;72(1):25-30.

17 Saito Y, Imai K, Nakamura R, Nanjo H, Terata K, Konno H, et al. Novel method for rapid in-situ hybridization of HER2 using non-contact alternating-current electric-field mixing. Sci Rep. 2016 Jul;6(1):30034.
18 Fujishima S, Imai K, Nakamura R, Nanjo H, Saito Y, Saito H, et al. Novel method for rapid fluorescence in-situ hybridization of ALK rearrangement using non-contact alternating current electric field mixing. Sci Rep. 2017 Nov;7(1):15116.

19 Kurihara N, Imai K, Nanjo H, Nakamura R, Wakamatsu Y, Akagami K, et al. Practical application of non-contact alternating current electric field mixing for reagent-saving in situ hybridisation of HER2. J Clin Pathol. 2019 Sep;72(9):603-8.

20 Jokoji R, Yamasaki T, Minami S, Komuta K, Sakamaki Y, Takeuchi K, et al. Combination of morphological feature analysis and immunohistochemistry is useful for screening of EML4-ALK-positive lung adenocarcinoma. J Clin Pathol. 2010 Dec;63(12):1066-70.

21 Seto T, Kiura K, Nishio M, Nakagawa K, Maemondo M, Inoue A, et al. CH5424802 (RO5424802) for patients with ALK-rearranged advanced non-small-cell lung cancer (AF-001JP study): a single-arm, open-label, phase 1-2 study. Lancet Oncol. 2013 Jun; 14(7):590-8.

22 Takamochi K, Takeuchi K, Hayashi T, Oh S, Suzuki K. A rational diagnostic algorithm for the identification of ALK rearrangement in lung cancer: a comprehensive study of surgically treated Japanese patients. PLoS One. 2013 Aug;8(8):e69794.

23 Takeuchi K, Togashi Y, Kamihara Y, Fukuyama T, Yoshioka H, Inoue A, et al. Prospective and clinical validation of ALK immunohistochemistry: results from the phase I/II study of alectinib for ALK-positive lung cancer (AF-001JP study). Ann Oncol. 2016 Jan; 27(1):185-92. 